\title{
Sensible reasoning in two tasks: Rule discovery and hypothesis evaluation
}

\author{
HILARY H. FARRIS and RUSSELL REVLIN \\ University of California, Santa Barbara, California
}

\begin{abstract}
The hypothesis testing skills of undergraduates were measured in two tasks: the 2-4-6 rule discovery task in which students generate and assess hypotheses, and a hypothesis evaluation task, which requires only the assessment of hypotheses. The results of Experiments 1 and 2 show that the students consistently employed a disconfirmation strategy when assessing hypotheses, but employed a counterfactual inference strategy when they also were required to generate the hypotheses. The results of Experiment 3 suggest that the selection of the hypothesis testing strategy reflected a balance between the logical requirements of the task and the desirability of possible outcomes. Taken together, the findings support a more consistent picture of human rationality across tasks, and suggest alternatives to accounts of confirmation bias.
\end{abstract}

Hypothesis testing is an intrinsic component of the scientific process. It is peculiar then-if not alarmingthat the hypothesis testing strategies of scientists as well as students have been characterized as biased and uncritical (e.g., Mahoney \& DeMonbreun, 1977; Mitroff, 1974; Tschirgi, 1980; Tweney et al., 1980; Wason, 1960). For example, in a rule discovery task developed by Wason $(1960,1968)$, students and scientists appear to seek confirmation of their hypotheses rather than a critical test of them. The picture is not completely negative, however. Students in some conditions of a hypothesis evaluation task (Tschirgi, 1980) do apply standard experimental procedures to critically test a hypothesis under consideration. These disparate findings render problematic the true nature of people's ability to reason "scientifically."

In the present study, we seek to mitigate these views of human reasoning by examining the situational and cognitive components of the rule discovery and evaluation tasks. In so doing, we will consider an alternative account that claims that reasoners follow a valid hypothesis testing process called counterfactual reasoning. This procedure superficially resembles the confirmation bias that so often has been used to characterize alogical thinking.

\section{RULE DISCOVERY}

One form of scientific reasoning occurs when we are asked to evaluate hypotheses for which there is a "true" rule to discover. The hypothesis testing procedures used by students in a rule discovery task were examined by Wason $(1960,1962,1968)$, who asked students to discover an arithmetic rule that the experimenter had in mind. The students were given a "seed" triple of numbers (e.g.,

Correspondence may be sent to Hilary Farris, Psychology Department, University of California, Santa Barbara, CA 93106.
$2,4,6)$ to begin the task and were told that the triple conformed to the rule. They were asked to evaluate their hypotheses by generating triples of numbers. The experimenter would provide feedback on whether the triple was consistent with the rule in question. This task is analogous to one faced by scientists, with the seed triple functioning as an initiating observation, and the act of generating the triple is equivalent to performing an experiment. The methodology is an extension of the work of Bruner, Goodnow, and Austin (1956).

While there are a number of ways in which the critical evaluation of hypotheses can be carried out, Wason focused primarily on the disconfirmation strategy in which the hypothesis tester generates counterexamples of the hypotheses under consideration. The formal basis for the strategy is as follows: (1) If hypothesis $H 1$ is true, then triple $T 1$ is true and $T 2$ is false; and (2) if $T 2$ is true, then $H 1$ is false. However, if the reasoner follows a confirmation strategy, then a third rule would be in effect: (3) If $T 1$ is true, then $H 1$ is true. Of course, this is a log ically unsound rule, because the most you can conclude from observing that $T 1$ is true is that $H 1$ is plausible.

The use of disconfirmation is operationalized in Table 1. Students following this rule of inference should seek to "falsify" their hypothesis by generating triples that would be false if their hypothesis is true (as with rule 2 above). This is in keeping with the philosophical notion of falsifiability and the critical testing of hypotheses (e.g., Popper, 1972).

Studies examining adult reasoning in Wason's 2-4-6 rule discovery task have been concerned primarily with extensions of the discovery paradigm in various environments (Gorman, Gorman, Latta, \& Cunningham, 1984; Gorman, Stafford, \& Gorman, 1987; Mahoney \& DeMonbreun, 1977; Mynatt, Doherty, \& Tweney, 1977, 1978). Other studies have been directed to facilitate students' use of disconfirmation within the Popperian framework of falsification (e.g., Gorman, 1986; Gorman \& 
Gorman, 1984; Tukey, 1986; Tweney et al., 1980; Wetherick, 1962). In general, these studies concur with Wason's analysis: A majority of individuals fail to consider alternate hypotheses and, instead, seek to confirm, by enumeration, a favorite hypothesis. This phenomenon in rule discovery is referred to as confirmation bias.

It should be noted that the correct solution does not absolutely require that the disconfirmation strategy be followed (Tukey, 1986; Tweney et al., 1980). For example, there are other rational strategies that one may employ in rule discovery other than disconfirmation (see a Bayesian analysis by Baron, 1985; the positive test strategy of Klayman \& $\mathrm{Ha}, 1987$; as well as the scholarly analyses by Hardin, 1980, and Tukey, 1986).

We present evidence here to suggest the presence of a specific rational strategy, counterfactual reasoning, that is part of the hypothesis testing process (Rescher, 1964; Revlis \& Hayes, 1972). When we follow this procedure, we consider the truth of a hypothesis by assuming it is false and observing the results. There are many derived forms of such reasoning, including reductio ad absurdum arguments in which one assumes that the antithesis of a to-be-proven argument is correct and evaluates the consequences (see, also, applications of counterfactual inference to language processing by Hornby, 1974).

The steps a student using this strategy might follow in the rule discovery task are shown in Table 1 and are illustrated by the following example. Suppose we believe that the rule the experimenter has in mind is "even numbers;"' this is tested by assuming that the hypothesized rule is false and positing, for example, that "odd numbers" is true. We then generate a triple that is consistent with the latter hypothesis (e.g., 3, 5, 7). If the experimenter confirms that the triple is consistent with the experimenter's rule, then "even numbers" is indeed false. If the triple is inconsistent with the rule, then "odd numbers" is false and "even numbers" is plausible. Notice that while the triple generated by the hypothesis tester may confirm the stated hypothesis, it actually disconfirms the target hypothesis that is assumed to be false. Unfortunately, if the reasoner follows the counterfactual

Table 1

Two Reasoning Strategies

Disconfirmation

Assume: Hypothesis $A$ is correct

Generate: Prediction (triple) that is inconsistent with $A$

Evaluate Feedback:

(a) If "Yes", then assumption about $A$ is incorrect

(b) If "No", then $A$ is likely to be correct

Counterfactual Reasoning

Assume: Hypothesis $A$ is incorrect

Select: Complementary hypothesis, $A^{\prime}$

Generate: Prediction (triple) consistent with $A^{\prime}$

Evaluate Feedback:

(a) If "Yes", then assumption about $A$ is correct

(b) If "Yes", then $A$ ' is plausible

(c) If "No", then $A$ is likely to be correct (where $A$ ' was a unique complement to $A$ ) strategy, the substantial frequency of confirming triples can be misinterpreted by the experimenter as evidence for a bias to confirm.

In the present study, we examine the possibility that students may engage in such forms of counterfactual inference during the rule discovery task and that the resulting behaviors may appear to be confirmation, when in fact they reflect sensible hypothesis testing strategies.

To evaluate the possibility that reasoners employ the counterfactual strategy, we must depart somewhat from the traditional paradigm. The statistical analyses in previous studies of rule discovery have tested whether the student-generated instances were consistent with previous hypotheses. In contrast, we are concerned with whether the hypotheses themselves are compatible with prior hypotheses. This approach gives us a more complete picture of the entire hypothesis testing strategy, because it provides an opportunity to distinguish between students using disconfirmation and those using counterfactual inference at the local level of a single statement of a hypothesis with its companion triple.

\section{HYPOTHESIS EVALUATION}

Decisions on hypothesis evaluation tasks provide a more optimistic picture of reasoning strategies. Tschirgi (1980) presented students with vignettes in which a story character had to test a hypothesis about the importance of one of three variables for an event in the story (see Appendix A). The students' task was to decide how the character should assess the hypothesis. There were three basic operations available to the reasoner: (1) Vary one thing at a time (called VOTAT), where the critical variable is manipulated; (2) hold one thing at a time (HOTAT), where the critical variable is not changed but all others are; and (3) change all variables. The VOTAT decision corresponds to disconfirmation strategy, while HOTAT corresponds to confirmation. Overall, adults preferred the disconfirmation (VOTAT) strategy. However, this preference was influenced by the desirability of the story outcome: When the outcome of the vignette was negative (as in Appendix A), adults followed the VOTAT (disconfirmation) strategy; when the outcome was positive (e.g., when the job interview went well), they followed the HOTAT (confirmation) strategy.

The apparent discrepancy in rationality between the rule discovery and hypothesis evaluation tasks may be attributable to the different demands they make on the reasoner. In rule discovery, the decisions reflect two components: (1) generation of hypotheses and (2) assessment of hypotheses (e.g., Weimer, 1977; also see Baron, 1985). The evaluation task, on the other hand, primarily requires assessment, since the hypotheses are provided.

We conjecture that the apparent confirmation bias in hypothesis testing cited by Wason and others may reflect the generation process. In rule discovery, the reasoner must generate and assess hypotheses culled from a potentially infinite set of hypotheses. One way to reduce the 
enormity of the task is to perform what Platt (1964) called strong inference and test multiple, competing hypotheses. The counterfactual reasoning strategy could be construed as a restricted form of strong inference, for which a single hypothesis and its competitor are considered.

In hypothesis evaluation, the hypothesis tester has to assess a single hypothesis sampled from a small set whose outcomes have differential desirability. Since this task primarily requires assessment, students use a strategy consistent with test-outcome utility: they use VOTAT when costs for disconfirmation are low (or benefits are high) and HOTAT when costs for disconfirmation are high (or benefits are low). This desirability function is analogous to scientists selecting to affirm pet hypotheses while seeking to disconfirm (falsify) competing ones. Here, we suggest that the strategies reflect not only task demands but additional contextual elements, such as the utility of holding or varying critical elements (see Baron, 1985).

This account anticipates that the sequence of hypotheses in the rule discovery task reflects a systematic counterfactual inference strategy of generating competing hypotheses that are assessed by means of confirming triples. To examine this claim, in Experiment 1, we assessed the relative frequency of confirmation and disconfirmation and traced the decisions of students across both tasks to determine whether the reasoners' strategies were consistent with a confirmatory or a counterfactual strategy. In Experiments 2 and 3, we looked more closely at the contextual nature of the strategies found in the hypothesis evaluation task and sought clarification of the outcome desirability function.

\section{EXPERIMENT 1}

This experiment contrasted rule discovery and hypothesis evaluation in a within-subjects design to examine individual differences in the use of two hypothesis testing strategies: disconfirmation and counterfactual reasoning.

\section{Method}

\section{Subjects}

Ninety-one undergraduates, enrolled in an introductory psychology course at the University of California at Santa Barbara, participated in Experiment 1 to meet a class requirement.

\section{Materials}

Rule discovery task. The students were provided with a record sheet to keep track of their hypotheses, number triples, and the experimenter's feedback. It contained four column headings: "Hypotheses,"' "Numbers," "Conforms," and "Does not Conform." The first line contained "Beginning Observation" in the Hypothesis column, ' 246 ' in the Numbers column, and a check mark in the Conforms column. The rule to be discovered was "numbers in ascending order." There were two sets of instructions, one that replicated instructions used by Wason (1960) and another, more explicit, version. These instructions are shown in Appendix B.

Hypothesis evaluation task. Twelve story problems were created using familiar, everyday situations: baking a cake (after Tschirgi, 1980), getting to class on time, planning a vegetable garden, training a dog, editing a newspaper, applying for a job, gambling, tak- ing a vacation, repairing a car, hanging wallpaper, writing a term paper, and doing a chemistry experiment. Appendix A illustrates a sample story problem.

Each story contained three unrelated variables that the story character manipulated, producing either a negative or a positive story outcome. The story character then proposed a hypothesis about which of these variables had produced the outcome. The question for the student was, "What should the story character do to prove his/her point?'” (Tschirgi, 1980)

Three possible actions were listed in a multiple-choice format at the end of the story. These actions represented the three strategies discussed earlier: the disconfirmation strategy (VOTAT), the confirming strategy (HOTAT), and the change-all strategy (CA). The VOTAT decision was the logically prescribed strategy in all of the problems, regardless of outcome.

To balance the stories and the positive/negative outcomes across problems, two different problem booklets were developed. Each booklet had the same stories in the same order, but with opposite outcomes (i.e., if a story in Booklet 1 had a positive outcome, it had a negative outcome in Booklet 2). The six positive outcome and six negative outcome stories in each booklet were randomly ordered.

\section{Design and Procedure}

There were four groups required by the orthogonal pairing of the two factors, task order (rule discovery and hypothesis evaluation) and instructions (replication and explicit). The students were run in groups of 4 , in sessions lasting approximately $60 \mathrm{~min}$.

Rule discovery task. The students in Groups 1 and 3 were given the replication instructions (Wason, 1960). Group 1 solved rule discovery first and Group 3 solved hypothesis evaluation first. Consistent with the original conditions, these groups were allowed to state as many incorrect rules as necessary for them to eventually arrive at the correct rule, or until $30 \mathrm{~min}$ had elapsed. The students who were incorrect were shown a written notice: "That is not the rule. Please continue."

The original (replication) instructions might have induced students to equate "high confidence" in a hypothesis with "proof" of it (noted by Wason, 1968). To examine this possibility, more explicit instructions were created. These instructions emphasized that the rule was an arbitrary rule from an infinite set and that the rule must be proved. Groups 2 and 4 operated under the explicit instructions. Group 2 worked on the rule discovery task first, while Group 4 solved the hypothesis evaluation task first. In contrast to the replication condition, the rule discovery task for Groups 2 and 4 ended with a student's first announcement of a rule, similar to the procedure of Gorman and Gorman (1984).

There were three steps in each trial: (1) each student wrote down a hypothesis, (2) he/she then generated a triple, and (3) each student received feedback from the experimenter. The stating of hypotheses just prior to generation of the triples provided an opportunity to collect concurrent verbalizations (Anderson, 1987; Ericsson \& Simon, 1980), while avoiding the problems of retrospective reports and rationalizations pointed out by Evans (1982).

Hypothesis evaluation task. This task was the same for all four groups, except for the variation in task order. The students received instructions similar to those given by Tschirgi (1980), except that the requirement to pick the action that was the most sensible was omitted.

Our instructions were as follows:

In this task, we are interested in how people go about solving problems in everyday life. You will be reading twelve stories about students just like you involved in everyday situations.

In each story, the story character manipulates three elements in a situation to produce some outcome. He (or she) then develops a hypothesis about one of the three elements as the reason for the outcome. Your task is to help the story character prove his (or her) hypothesis. 
Pick the answer that will prove the point the story character wishes to make. Remember that the instruction to "prove" means to show that something is really true or really false.

\section{Results}

Experiment 1 had multiple objectives, and we will need to examine the results in three sections: (1) rule discovery, (2) hypothesis evaluation, and (3) individual differences across tasks. As we examine the decisions in the rule discovery task, we will alternately consider two points of view: (1) the traditional, falsification approach (e.g., Wason, 1962), which claims that the optimum method of disconfirmation occurs only when the number triples (i.e., tests) are generated to be incompatible with the hypothesis under consideration, and (2) the counterfactual reasoning strategy, which claims that a hypothesis may be disconfirmed by confirming a competing hypothesis.

\section{Rule Discovery: Traditional Analysis}

The students and procedures produced success rates that are comparable to those of Wason (1960). The proportion of students who were correct on their first or subsequent announcements of a rule are given for all groups in Table 2. This table shows that the groups met or exceeded the success rates shown in previous studies (Wason, 1960: $21.4 \%$ for first rule announcement and $75 \%$ for subsequent announcement). The proportion of solvers was equivalent across all groups and conditions.

Wason (1960) found significant differences between subjects who were correct on their first announcement of the rule and those who were incorrect, in terms of three dependent measures: (1) the total number of trials generated, (2) the proportion of triples that resulted in negative feedback (i.e., negative instances of the rule), and (3) an eliminative/enumerative index (a ratio of the number of triples incompatible with stated hypotheses to the total number of triples compatible with the stated hypotheses). A comparison of correct and incorrect first solvers on each of these measures is presented in Table 3, which shows that the present study replicated the typical findings (e.g., Mahoney \& DeMonbreun, 1977; Wason,

Table 2

Percentage of Students Correctly Announcing a Rule Groups

\begin{tabular}{lcccc}
\cline { 2 - 4 } \multicolumn{1}{c}{ Rule } & $\begin{array}{c}\text { Replication 1 } \\
(n=23)\end{array}$ & $\begin{array}{c}\text { Explicit 2 } \\
(n=22)\end{array}$ & $\begin{array}{c}\text { Replication 3 } \\
(n=18)\end{array}$ & $\begin{array}{c}\text { Explicit 4 } \\
(n=21)\end{array}$ \\
\hline First & 39.1 & 40.9 & 33.3 & 38.1 \\
Subsequent & 73.9 & & 75.0 & \\
\hline
\end{tabular}

Table 3

Three Measures of Reasoning Effectiveness

\begin{tabular}{|c|c|c|c|c|}
\hline \multirow[b]{2}{*}{ Measure } & \multicolumn{2}{|c|}{$\begin{array}{c}\text { Correct Rule } \\
(n=32)\end{array}$} & \multicolumn{2}{|c|}{$\begin{array}{l}\text { Incorrect Rule } \\
(n=52)\end{array}$} \\
\hline & $M$ & $S D$ & $M$ & $S D$ \\
\hline Number of Trials & 9.8 & 3.6 & 6.4 & 4.9 \\
\hline Percent Negative Instances & 28.8 & 10.7 & 7.9 & 13.5 \\
\hline Eliminative/Enumerative Index & 1.6 & 0.63 & 0.5 & 0.58 \\
\hline
\end{tabular}

Table 4

Percent Trials with "No" Feedback

\begin{tabular}{ccrrrr} 
& \multicolumn{2}{c}{ Correct Rule } & & \multicolumn{2}{c}{ Incorrect Rule } \\
\cline { 2 - 3 } \cline { 5 - 6 } Groups & $M$ & $S D$ & & $M$ & $S D$ \\
\hline Replication & 30.3 & 9.5 & & 3.3 & 8.1 \\
Explicit & 27.4 & 11.8 & & 12.5 & 16.1 \\
\hline
\end{tabular}

1960). First, students who were correct on their first announcement generated approximately 53\% more trials prior to their announcement than did those who were incorrect $[F(1,76)=10.4, p<.01]$. This productivity measure did not interact with instructions or task order.

Second, the correct students also generated a greater percentage of tests with negative feedback than did those who were incorrect $[F(1,76)=58.2, p<.01]$. Indeed, every student correct on their first rule had at least one "No" from the experimenter. Table 4 shows that the incorrect students receiving the explicit instructions had a slightly greater rate of negative instances than did those following the replication instructions; this contributes to an interaction between rule success and instructions $[F(1,76)=4.9, p<.05]$. The correct solvers generated this type of negative feedback from the experimenter four times more often than did the students who were incorrect. However, such negative feedback occurred on only $30 \%$ of the trials. If such feedback was deliberately elicited by the students, it still leaves the motivation for $70 \%$ of the responses unaccounted for.

The proportion of negative instances was not diagnostic for success on the second announcement of a rule. Of the 24 students in Groups 1 and 3 who were incorrect on their first rule, there was no significant difference in the proportion of negative instances between those whose second announcements were correct $(n=4)$ and those whose were incorrect $(n=20)$. Both groups produced negative instances on approximately $24 \%$ of the trials between the first and second announcements.

Negative feedback per se does not guarantee that reasoners are using a disconfirming strategy, only that they have selected a triple of numbers that is inconsistent with the experimenter's rule. To assess the overall tendency to disconfirm, Wason (1960) developed an eliminative/enumerative index, which we duplicate here. For each stated hypothesis, the current, and all subsequent, triples were coded as either compatible or incompatible with that hypothesis. A ratio of the total number of incompatible triples to the total number of compatible ones generated prior to the first announcement of a rule was computed for each subject. Table 3 shows that successful students had a higher eliminative/enumerative index than did unsuccessful ones $[F(1,76)=55.5, p<.01]$, with no main effect or interactions for instructions or task order. This index similarly distinguishes between solvers and nonsolvers on the second announcement of a rule $[F(1,21)$ $=6.09, p<.05]$.

The eliminative/enumerative index represents a global view of eliminative thinking, in which triples are checked against all previous hypotheses. As such, the index does 
not distinguish between students using disconfirmation and those using counterfactual inference at the local level of a single statement of a hypothesis with its companion triple. The next analysis provides a closer inspection of students' hypothesis testing strategies at this local level.

The paradigmatic example of disconfirmation is where a generated triple is incompatible with its accompanying hypothesis. To assess this direct, local indication of disconfirmation, we classified whether a triple was compatible or incompatible with the reason given for that triplethis measure is termed TR. A ratio of the total number of incompatible TRs to the total number of compatible ones (up to the first rule announcement) was computed for each student.

For all groups, this index fails to distinguish between those students who were correct on their first announcement of a rule and those who were incorrect (for replication groups, correct $=0.10$ and incorrect $=0.01$; for explicit groups, correct $=0.11$ and incorrect $=0.06$ ) . It also does not distinguish between those who were correct or incorrect on their second rule announcement (correct $=0.0$ and incorrect $=0.15$ ). Notice that this index, which instantiates the prescribed method of disconfirmation (taught to subjects in Gorman, 1986; Gorman \& Gorman, 1984; Gorman et al., 1987; Tweney et al., 1980), is not sufficient to explain individual differences related to successful solution in Experiment 1.

Furthermore, use of disconfirmation does not appear to be necessary for the successful testing of hypotheses (e.g., Tukey, 1986). Seventy percent of the firstannouncement solvers in Experiment 1 did not show a single instance of a triple that was incompatible with its stated hypothesis. These successful students only generated triples compatible with their hypotheses.

Not only is success in this task not dependent upon the use of disconfirmation, the absence of disconfirmation (i.e., the use of confirmation) does not guarantee failure. While $81 \%$ of the students never generated a triple incompatible with its stated hypothesis, $32 \%$ of these students were successful on their first announcement of a rule. In contrast, only $19 \%$ of the subjects used direct disconfirmation; of these, $65 \%$ were correct on their first rule announcement. Clearly, the use of disconfirmation is sufficient, but not necessary, for successful rule discovery on this task.

\section{Rule Discovery: Counterfactual Reasoning Analysis}

Under the counterfactual reasoning strategy, one hypothesis and its confirming triple can jointly disconfirm an incompatible hypothesis. To determine whether students were following this strategy, two variables were considered. The first coded whether or not the student's stated reason (i.e., hypothesis) for generating a particular triple was incompatible with all previous reasons (this measure is called RR). The second variable (called TT) coded whether or not a triple was incompatible with all previously stated triples (as an independent check that al- ternate hypotheses were being considered). For each variable, on each trial, the student was given a 1 or a 0 , depending on whether the relationship in question was incompatible or not. The percentages of incompatible RRs and TTs (i.e., the measures of counterfactual reasoning) were computed for the trials prior to the first rule announcement. In a sense, this assessment implies that students "look back" over previous hypotheses to develop their current tests. While we can offer no independent evidence of such backward glances, this notion is part of other conceptions of the hypothesis testing process (e.g., Klahr \& Dunbar, 1988).

Table 5 shows that solvers had a greater percentage of trials with reasons that were incompatible with previous reasons $(\mathrm{RR})[F(1,76)=16.8, p<.01]$ and a greater percentage of trials with triples that were incompatible with previous triples (TT) $[F(1,76)=11.9, p<.01]$ than did nonsolvers. This was in contrast with the standard measure of disconfirmation, TR (triples incompatible with their stated reasons), which was unrelated to success $[F(1,76)=3.7$, n.s. $]$. No other significant main effects or interactions were observed.

The same dependent variables were used to assess the students' disconfirmation behavior in the replication conditions after they received feedback that their first rule announcement was incorrect. The students who subsequently announced a correct rule generated a greater percentage of RRs $[F(1,22)=7.9, p<.01]$ and TTs $[F(1,22)=4.7, p<.05]$ than did those who announced an incorrect rule. Once again, the measure of disconfirmation, TRs, did not distinguish between correct and incorrect second announcements of the rule $[F(1,22)=0.7$, n.s.]. In summary, for both the first and second rule announcements, the counterfactual reasoning measures (RR and TT) successfully distinguished between solvers and nonsolvers, whereas the disconfirmation measure (TR) did not.

Looking only at the replication groups, about $20 \%$ of the students who were incorrect on their first rule announcement were correct on their second. To what processes can this improvement be attributed? For all students who were incorrect on their first announcement of a rule, a difference score was obtained for each of the three hypothesis testing measures by subtracting the stu-

Table 5

Percent Disconfirmation and Counterfactual Reasoning

\begin{tabular}{|c|c|c|c|}
\hline \multirow[b]{2}{*}{ Subjects } & \multirow{2}{*}{$\frac{\text { Disconfirmation }}{\text { Percent TRs }}$} & \multicolumn{2}{|c|}{ Counterfactual Reasoning } \\
\hline & & Percent RRs & Percent TTs \\
\hline \multicolumn{4}{|c|}{ First Rule } \\
\hline Solvers $(n=32)$ & 6.8 & 37.9 & 65.4 \\
\hline Nonsolvers $(n=52)$ & 2.3 & 18.7 & 47.7 \\
\hline \multicolumn{4}{|c|}{ Second Rule } \\
\hline Solvers $(n=4)$ & 0.0 & 47.4 & 81.3 \\
\hline Nonsolvers $(n=20)$ & 11.4 & 13.7 & 37.3 \\
\hline
\end{tabular}

Note-TRs = triples incompatible with their stated reasons; RRs $=$ reasons incompatible with previous reasons; and TTs = triples incompatible with previous triples. 
Table 6

Difference in Disconfirmation and Counterfactual Measures After First Rule Incorrect

\begin{tabular}{lcccc}
\hline & \multicolumn{2}{c}{ Disconfirmation } & & \multicolumn{2}{c}{ Counterfactual Reasoning } \\
\cline { 5 - 6 } \cline { 5 - 6 } Subjects & TRs & & RRs & TTs \\
\hline Solvers $(n=4)$ & 0.0 & & 45.4 & 20.8 \\
Nonsolvers $(n=20)$ & 10.4 & & -1.8 & -13.3 \\
\hline
\end{tabular}

Note-TRs = triples incompatible with their stated reasons; RRs = rea sons incompatible with previous reasons; and TTs = triples incompatible with previous triples

dents' scores prior to the first rule announcement from their scores generated between the first and second rule announcements. The results are presented in Table 6, which shows that only the difference score for RRs successfully distinguishes between the two groups $[F(1,22)$ $=7.7, p<.01]$. The solvers increased their percentage of alternate hypotheses by about $45 \%$. In contrast, nonsolvers did not significantly change their hypothesis testing strategies.

\section{Discussion of Rule Discovery}

Using the traditional analysis of hypothesis testing in rule discovery, the present data generally replicate Wason's (1960) findings. While the overall proportion of solvers on the first announcement of the rule was typically low, at $38 \%$, they generated more trials and had a greater proportion of negative instances than did the students whose first announcements were incorrect. The solvers also scored significantly higher on a global measure of eliminative thinking, using Wason's eliminative/enumerative index.

An analysis at a more local level, that of a single hypothesis and its accompanying triple, shows that the measure of disconfirmation, TR, did not discriminate between solvers and nonsolvers, and only marginally correlated with successful hypothesis testing $(r=.19$, $p<.10$ ). In fact, $70 \%$ of the students who were correct on their first rule announcement never generated a triple that was incompatible with its stated hypothesis. This finding is inconsistent with conclusions drawn in previous studies (e.g., Mahoney \& DeMonbreun, 1977; Wason, 1960 ) that a method of disconfirmation using this form of counterexample would be the preferred method of solvers. Measures of a counterfactual reasoning strategy, however, can discriminate between successful and unsuccessful rule discovery. The percentage of RRs was the best single predictor of success on the first rule announcement $(r=.43, p<.01)$. This was followed by TTs $(r=.33, p<.01)$.

While the solvers considered alternate hypotheses (RRs) twice as often as did the students who were incorrect, the nonsolvers did consider alternatives in almost $20 \%$ of their "experiments" prior to their first announcement. This contradicts the notion that students who are unsuccessful on the first rule announcement fail to consider alternate hypotheses. Of these initial nonsolvers, those who announced a correct rule on their second attempt increased their percentage of incompatible alternate hypotheses by $45 \%$. In a sense, these students (although few in number in the present study) increased their use of counterfactual reasoning after learning that their first rule was incorrect.

This is not to suggest that simple instructions to consider multiple hypotheses will be an effective means to increase student accuracy. Tweney et al. (1980) asked students to generate sets of two hypotheses together with two triples and found no improvement in accuracy. Baron (1985) notes that these students appear to generate dummy alternatives rather than the kind of competing hypotheses that our students seemed to generate spontaneously.

Based on the protocols provided by all students, successful ones, as well as half of the nonsolvers, appear to employ a strategy consistent with counterfactual reasoning rather than direct disconfirmation. This suggests an additional reason why the explicit instructions in the present study and previous efforts to instruct students to use disconfirmation have been ineffective (Tweney et al., 1980 ) or marginally effective (Gorman, 1986; Gorman \& Gorman, 1984; Gorman et al., 1987). Since students are already employing a sensible strategy (namely, counterfactual reasoning), instructions to generate counterexamples may be unnecessary at best.

One procedure previously shown to result in improved success on rule discovery fits well with this analysis. Tweney et al. (1980) told students that they were to discover two rules, DAX and MED. Successful subjects typically sought confirming evidence alternating between the two rules. Similarly, the students in Experiment 1 appeared quite naturally to relinquish confirmed hypotheses to seek confirmation of incompatible, alternate hypotheses (see also Klahr \& Dunbar, 1988). While the solution rate in the DAX/MED study was higher than that of the present one (see also Gorman et al., 1987; Tukey, 1986), the success of the manipulation might be attributed to providing a framework that is compatible with a strategy that students already implicitly employ.

Even if students are following strategies other than counterfactual reasoning, there are ample proposals for an underlying rationality to their judgments. For example, Tukey (1986) suggests at least three possible "logics" that could be followed by students. Klayman and Ha (1987) propose a positive test strategy based on the informativeness of a confirming test of a "minority phenomenon"' (see also Bowers, 1977). Students operating within these conceptions of the task would produce a generation of triples similar to those commonly observed.

One implication of the findings in Experiment 1 is that, in assessing hypothesis testing, if rationality is limited to the generation of counterexamples (i.e., disconfirmation), it will tend to underestimate the total amount of critical testing and overestimate the amount of confirmation bias in rule discovery. We turn now to an analysis of decisions reached by the same students in a hypothesis evaluation task. 


\section{Hypothesis Evaluation}

Previous findings on the hypothesis evaluation task (Tschirgi, 1980) suggest that students will generally employ a disconfirmation strategy (VOTAT), but that they do so more often when the story outcome is undesirable than when it is desirable. The data from Experiment 1 are given in Table 7, which shows that students chose the VOTAT response on positive and negative outcome stories more often than would have been expected by chance (positive outcome: $56 \%$ of the time, $p<.1$; negative outcome: $86 \%, p<.01$ ). The preference for disconfirmation was greater on negative outcome vignettes than on positive ones $[F(1,78)=70.7, p<.01]$. This preference was unaffected by task order, rule instructions, or booklet.

Hypothesis evaluation styles. To examine individual differences in hypothesis testing, each student was placed in one of five style categories: (1) Perfect VOTAT, these are students who chose all 12 VOTAT responses $[X=12$, $p<.01]$. Of the 84 students in the final data set, $11.9 \%$ $(n=10)$ made VOTAT decisions on all 12 problems. (2) VOTAT, these are students who chose at least 9 and no more than 11 VOTAT responses out of 12 answers $[12 \geq X \geq 9, p<.01] ; 42.9 \%$ of the students $(n=36)$ fell into this category. (3) HOTAT, these chose at least 9 HOTAT responses out of 12 answers $[X \geq 9$, $p<.01$ ]; $2.3 \%$ of the students $(n=2)$ were in this category. (4) Context-dependent, these students chose at least four VOTAT responses on six negative outcome problems $[6 \geq X \geq 4, p<.01]$ and at least four HOTAT responses on six positive outcome problems; $27.4 \%$ of the students $(n=23)$ conformed to this style. (5) Other, the students were classified as "other" if their hypothesis testing did not fit any of the four classifications above; $15.5 \%$ of our sample were unclassified $(n=13)$.

These categories make clear that a majority of the students employed the normative strategy: approximately $55 \%$ of the students (those classified as VOTAT or Perfect VOTAT) could be considered logical reasoners, employing disconfirmation even on positive outcome problems. In contrast, the strictly Context-dependent reasoners made up less than one-third of the students. Even with the HOTATs added in, less than one-third of the students (30\%) showed confirmation bias by choosing the confirming HOTAT option in positive outcome situations.

Table 7

Percent VOTAT Decisions for Two Outcomes

\begin{tabular}{lccc} 
& \multicolumn{2}{c}{ Groups } & Tschirgi \\
\cline { 2 - 3 } Outcomes & Replication & Explicit & $(1980)$ \\
\hline Positive & 59.5 & 52.5 & 35.0 \\
Negative & 89.7 & 81.4 & 75.0 \\
\hline
\end{tabular}

Note-VOTAT $=$ vary one thing at a time.

\section{Individual Differences Across Tasks}

While the style variable helps us to see the dominant response profiles in the hypothesis evaluation task, style was unpredictive of the strategies used by reasoners in rule discovery. There was no significant difference among these groups in the frequency of correct first rule announcements in the rule discovery task. For example, only 4 of the 10 subjects who scored Perfect VOTATs on the evaluation task were correct on their first announcement of a rule. Only 1 of the 2 HOTAT subjects was incorrect.

Consequently, we considered the predictive ability of two other variables: (1) the overall percentage of VOTAT decisions on the 12 stories, and (2) the percentage of VOTAT decisions on only the six positive outcome stories. Each student's VOTAT scores on these variables were correlated with their scores on four rule discovery measures: RR, TT, TR, and Wason's eliminative/enumerative index. The students were grouped according to whether or not they were correct on their first rule announcement

For the students who were correct on their first announcement of a rule, the strategies used in rule discovery bear little relationship to those evoked by hypothesis evaluation. On all 12 problems, the percentage of VOTAT decisions was positively correlated with the counterfactual reasoning measure (RR: $r=.34, p=.05$ ), but negatively correlated with the disconfirmation strategy (TR: $r=-.38, p<.05$ ). Of special interest is the relation between decisions on rule discovery and hypothesis evaluation on the six positive outcome problems. Table 8 shows that $97 \%$ of the students who announced the correct rule on the first occasion used some form of counterfactual reasoning (RR $>0 \%$ ); of these, $71 \%$ used the disconfirmation strategy (VOTAT $\geq 50 \%$ ) in evaluating hypotheses on the positive outcome problems. The only student who used a pure disconfirmation strategy in rule discovery used a confirmation strategy in hypothesis evaluation.

For the students who were incorrect in their first announcement of a rule, there was a negative correlation between disconfirmation in hypothesis evaluation and Wason's eliminative thinking in rule discovery (overall VOTAT: $r=-.36, p<.01$; positive VOTAT: $r=-.33, p<.05$ ). Otherwise, no relation between strategies on the two tasks was discernible. Table 8 shows that only 6 students were confirmers on both tasks, and that, independent of the strategy employed in rule discovery, most students (64\%) followed a disconfirmation strategy in hypothesis evaluation.

\section{Discussion of Hypothesis Evaluation}

Disconfirmation is the dominant strategy in hypothesis evaluation: less than one-third of the students in Experiment 1 could be characterized as possessing anything equivalent to a bias to confirm. In addition to disconfirmation, the students' decisions reflected outcome desira- 
Table 8

Frequency of Reasoners Using Different Strategies

\begin{tabular}{|c|c|c|c|}
\hline \multirow[b]{2}{*}{ Rule Discovery } & \multicolumn{3}{|c|}{$\begin{array}{l}\text { Hypothesis Evaluation: } \\
\text { Positive Outcome Problems }\end{array}$} \\
\hline & $\begin{array}{c}\text { Disconfirming } \\
\text { (VOTAT } \geq 50 \% \text { ) }\end{array}$ & $\begin{array}{c}\text { Confirming } \\
\text { (VOTAT }<50 \%)\end{array}$ & Total \\
\hline \multicolumn{4}{|c|}{$\begin{array}{ll}\text { First Rule Correct } \\
\end{array}$} \\
\hline Counterfactual $(\mathrm{RR}>0 \%, \mathrm{TR}=0 \%)$ & 15 & 7 & 22 \\
\hline Disconfirming ( $R R=0 \%, T R>0 \%)$ & 0 & 1 & 1 \\
\hline Mixed $(\mathbf{R R}>0 \%, \mathrm{TR}>0 \%)$ & 7 & 2 & 9 \\
\hline Confirming $(\mathrm{RR}=0 \%, \mathrm{TR}=0 \%)$ & 0 & 0 & 0 \\
\hline \multicolumn{4}{|c|}{ First Rule Incorrect } \\
\hline Counterfactual & 15 & 10 & 25 \\
\hline Disconfirming & 1 & 1 & 2 \\
\hline Mixed & 2 & 2 & 4 \\
\hline Confirming & 15 & 6 & 21 \\
\hline
\end{tabular}

Note-VOTAT $=$ vary one thing at a time.

bility, since they wished to preserve or obtain a desirable outcome, as well as a definitive test (e.g., Baron, 1985). But the former was subservient to the interests of the latter, since students selected the logical test more often than chance for every vignette.

\section{Discussion}

These findings call into question the notion of confirmation bias. If such a bias existed, it should have been an inherent source of decisions across tasks. Yet, only $7 \%$ of the students could be said to have used such a bias for making a decision. Even if all of the counterfactual inferences were recategorized as confirmations, less than one-third of the students would have been consistent across the two tasks (see Table 8).

At first glance, there appears to be little correspondence between the strategies used in the two tasks. While the students appreciated the logical value of direct disconfirmation in hypothesis evaluation, they followed a counterfactual reasoning procedure in rule discovery. However, this was to be expected, because, as Weimer (1977) has indicated, rule discovery tasks require the additional processes of generating and sampling from a hypothesis set along with the assessment required by both tasks. Since the hypothesis set in the present rule discovery task was unlimited, such a strategy as counterfactual reasoning was an appropriate one to follow, because it allowed for the critical testing of competing hypotheses. Consequently, the overall strategy in the two tasks corresponded to a rational tactic of balancing the dual goals of assessment and desirability in the evaluation task, and assessment and efficiency in the discovery task.

Of course, it can be claimed that the evaluation task was so restricted that the students were not free to select attractive, but nonrational, alternatives, or that outcome desirability, as a variable, was too constrained. We turn to these questions in Experiments 2 and 3.

\section{EXPERIMENT 2}

The hypothesis evaluation task in Experiment 1 might not have provided the reasoners with a plausible assessment problem. The task required only that the students select from three constrained response options, while the three variables to be manipulated by the story character could actually generate eight possible tests: one VOTAT and one HOTAT test for each of the three variables, one change-all, and one hold-all (i.e., keep everything the same). Consequently, the evaluation task might have unnecessarily restricted the students' hypothesis testing options and evoked quite different reasoning strategies than did the rule discovery task. To examine this possibility, Experiment 2 offered the reasoner all possible manipulations of the story variables and made the evaluation task more of a production task.

\section{Method}

\section{Subjects}

Sixty students were given the same booklet and instructions as those used in Experiment 1, with the exception of a change in the answer format. Experiment 2 was conducted in a single session, lasting $20 \mathrm{~min}$.

\section{Procedure}

The format change required the students to indicate whether or not each variable mentioned in the vignette was to be kept the same or changed in the test of the hypothesis. This required students to construct one of the eight possible configurations of variables for testing. Appendix $\mathrm{C}$ shows a sample problem with this new format.

\section{Results}

The students were categorized as adopting one of six strategies on each problem, on the basis of their manipulations of the three elements in each story. The strategies were: (1) Hold-all ("keep the same" option selected for each variable); (2) VOTAT ("change" selected for the 
critical element and "keep" for the other two elements); (3) XVOTAT ("change" selected for a noncritical element, while "keep" selected for the other elements); (4) HOTAT ("keep the same" option for the critical element and "change" option for the other two); (5) XHOTAT (HOTAT on a noncritical element only); and

(6) Change-all ("change"' for all elements).

This detailed categorization reveals that the three options provided in Experiment 1 appear to be only the set that the students considered relevant. Less than $14 \%$ of the 60 students selected options other than the three hypothesis tests previously offered. These other strategies reflected $3.1 \%$ of the hypothesis tests for positive outcome stories and $1.7 \%$ of the negative outcome stories. Overall, $65 \%$ of these alternative hypothesis tests were XVOTAT, a VOTAT strategy applied to a noncritical variable.

As a consequence of this distinct preference for only the three options used previously, the proportion of VOTAT decisions was the dependent variable of interest. To assess hypothesis testing with this full set of options, the percentages of logically correct VOTAT scores were compared with those of Groups 3 and 4 from Experiment 1 . They were used as a control, since the evaluation task was not preceded by the rule discovery task for these groups.

The proportions of disconfirmation judgments are presented in Table 9, which shows that the students' strategies were not affected by the opportunity to generate tests from the full set of possible manipulations. Overall, the students used the VOTAT strategy more often on negative outcome stories than they did on positive ones $[F(1,98)=49.8, p<.01]$, and this preference was not affected by the number of options the student had available.

\section{Discussion}

The results suggest that the students had a tacit grasp of the logical implications of direct disconfirmation; they were capable of spontaneously generating logical proofs with a finite set of hypothesis tests. While outcome desirability played a part in the selection of the type of test, the students maintained an overall disconfirmation strategy. This replicates the findings of Experiment 1, in which the students were faced with a more restricted set of alternatives. Still at issue, however, is the nature of this desirability function and how it contributes to the overall rationality of the reasoners' judgments.

Table 9

Percent Disconfirmation (VOTAT) Decisions In Two Kinds of Assessment

\begin{tabular}{lcc}
\hline & \multicolumn{2}{c}{ Outcome } \\
\cline { 2 - 3 } Option Format & Positive & Negative \\
\hline Full Set & 63.1 & 80.6 \\
Restricted Set & 54.0 & 84.1 \\
\hline
\end{tabular}

Note-VOTAT $=$ vary one thing at a time.

\section{EXPERIMENT 3}

In Experiments 1 and 2, outcome desirability appeared to exercise an important influence on the students' selection of variables to be tested. This was an effective tactic taken by the students, since it was natural for them to want the conditions that follow a definitive test to be as desirable as possible. So, for example, we not only want to isolate the critical factor in a job interview, we also want to get the job. If hypothesis evaluation bears any correspondence to the kind of hypothesis testing that we perform as scientists, then the desirability factor may be analogous to the process of selecting paradigms and variables so that the results will be interesting.

Desirability (and utility) are generally viewed as continuous variables. If they aptly reflect the reasoners' decision processes, then the application of the VOTAT strategy ought to be a continuous function of the desirability of the test outcome. Previously, only two levels of desirability, positive and negative, have been employedleaving the question of continuity problematic. Experiment 3 sought at least an initial examination of the desirability function.

It is difficult to create story problems with intermediate outcomes, since situations in the real world rarely are value-free. However, we sought to render some story problems more neutral than others by varying the reasoner's degree of involvement with the story character. If disconfirmation is a continuous function of desirability, then the mean VOTAT response for each story problem should be roughly inversely proportional to the degree of desirability as manipulated by involvement.

\section{Method}

\section{Subjects}

Twenty-two undergraduate psychology students were presented with the same story problems and instructions as in Experiment 1.

\section{Procedure}

To vary the degree of subject involvement with the story, a second story character was introduced in four of the problems. This character always offered advice on how to prove a point. Therefore, the answer selecton on these problems indirectly asked students to choose advice offered by the buffer character rather than to select the test to be performed. An example of a neutral, though slightly negative, outcome story is shown in Appendix D.

The outcomes for the twelve story problems in each booklet were positive (4), neutral-positive (2), neutral-negative (2), and negative (4) outcomes. Two booklets were created so that each story would appear in both outcomes across subjects (following the procedure of Experiment 1).

\section{Results}

The average VOTAT score was computed for each student on each of the four types of outcome problems. To assess the reliability of our findings, we compared the VOTAT scores for positive and negative outcome problems with the scores for the same problems in Experiment 1 . For positive outcomes, the average VOTAT 
was $53.5 \%$ and $50.0 \%$ for Experiments 1 and 3, respectively. For negative outcomes, the means were $85.6 \%$ and 87.5\% for Experiments 1 and 3, respectively. This comparability gives us some confidence in assessing the importance of outcome desirability over the entire range of values in Experiment 3.

The VOTAT scores across conditions are summarized in Table 10, which shows that the students selected more critical tests as problem outcome became more negative $[F(3,60)=7.5, p<.01]$. There was a strong linear trend $[F(1,2)=450, p<.01]$, with an adjusted $R^{2}$ of 0.99 (estimated intercept $=38.1 \%$ ). Although 11 of the 22 students did not exhibit this precise ordering across all four outcome variables (12 possible paired comparisons), only 2 of these students made more disconfirming (VOTAT) decisions on positive outcomes than they did on negative ones. There was no main effect of booklet nor did it interact with problem outcome.

\section{Discussion}

These data suggest that the use of disconfirmation was a continuous function of event outcome desirability, although it was not the sole basis for judgments since, even in the most extreme situation, disconfirmation (VOTAT) was employed more often than would have been expected by chance.

The importance of desirability appeared to be related to the reasoner's involvement with the story events. When the students were evaluating advice, they adhered to the notion of disconfirmation more closely than when they had to carry out the hypothetical advice and suffer the test outcome. Perhaps we have observed, in Experiment 3, what we experience professionally: It is easier to adhere to the highest standards of research when we review the work of others than it is when we review our own. Not that our ethics are necessarily situational, but that we are more sensitive to the consequences of the study when they are proximal rather than distal.

\section{CONCLUSION}

The present study has sought to understand two conflicting views of adult hypothesis testing abilities by contrasting decisions in rule discovery and hypothesis evaluation tasks. Judgments in the former task have long been characterized as biased and uncritical, with adults apparently seeking to confirm hypotheses rather than to disconfirm them. In contrast, adult reasoners frequently use

Table 10

Percent VOTAT Decisions for Four Types of Outcomes

\begin{tabular}{lcc}
\hline \multicolumn{1}{c}{ Outcome } & $M$ & $S D$ \\
\hline Positive & 50.0 & 40.1 \\
Neutral-Positive & 63.6 & 38.4 \\
Neutral-Negative & 77.3 & 29.8 \\
Negative & 87.5 & 18.5 \\
\hline
\end{tabular}

Note-VOTAT $=$ vary one thing at a time. a disconfirming strategy, commonly held to be the standard for scientific thinking (but see Mahoney, 1979, 1980), to test hypotheses in the evaluation task. By having the students perform both tasks, it was possible to examine individual differences as well as the importance of task constraints.

When the students generated and tested hypotheses in the rule discovery task, they appeared to employ a form of counterfactual inference. They sought confirmation of alternate hypotheses that were incompatible with previously stated hypotheses. The degree to which the students engaged in counterfactual reasoning was the best single predictor of success in the task. In contrast, the prescribed form of disconfirmation (in which triples are generated to be incompatible with the current hypothesis) was used by a minority of students (31\%) to discover the rule. It was neither predictive of success in rule discovery nor was it correlated with disconfirmation in the hypothesis evaluation task. This accords with previous studies in which instructions to disconfirm did not improve accuracy, when compared with an appropriate control group (e.g., Gorman \& Gorman, 1984, Experiment 1).

Counterfactual inference bears a superficial resemblance to confirmation bias, since in both cases the reasoner will generate triples compatible with the stated hypothesis. It is not surprising then that prior research with rule discovery has characterized subjects as alogical (e.g., Mahoney \& DeMonbreun, 1977; Wason, 1960, $1962,1968)$. It is only when we consider the correspondence across hypotheses that we observe what Platt (1964) referred to as the working of a "strong inference" strategy. Tukey (1986) suggests similar possibilities when he discusses other forms of inference that might be used in the task. This is related to the broader issue of what kind of logics might generate apparently irrational, as well as rational, decisions (see Cohen, 1981)-questions that we make no effort to resolve with the present study.

If students' decisions are governed by some internal psychologic, as suggested here and by others (e.g., Klayman \& Ha, 1987; Tukey, 1986), then whence comes failure? We present evidence here that initial nonsolvers in rule discovery fail to perform enough experiments. Or, as Baron (1985) has suggested, they simply stop too soon because of their high confidence in their current hypothesis and consequent lack of utility of additional tests. In addition, unsuccessful students consider proportionally fewer contradictory hypotheses. That is, they differ in the extent to which they use counterfactual reasoning. Indeed, the single factor that characterizes the ultimate success of these students is the increase in the proportion of counterfactual tests that they generate after their first incorrect announcement of a rule.

Decisions in the hypothesis evaluation task reflect not only the logical requirements of the task, but outcome desirability as well. When the current state of affairs is undesirable (e.g., the job interview was not successful), students readily manipulate the critical variable and hold all others constant. If the test then supports the control- 
ling hypothesis, they will benefit from also having produced a desirable state of affairs (a successful interview). When the initial conditions are positive (e.g., the interview was already successful), students tend to hold the critical variable constant and manipulate all others so that a favorable test will also produce a desirable final state of affairs. This is consistent with Baron's analysis that outcome utility shapes the tests that the reasoners employ. However, in our present study, desirability rarely controlled a student's overall decisions: two-thirds of all students used a disconfirming strategy across problem outcomes.

The degree to which disconfirmation is employed in evaluating hypotheses is related to the reasoner's involvement with the event's outcome. When students are evaluating the advice offered by others, they employ a disconfirmation strategy more often than when they provide the advice themselves and have to accept undesirable test outcomes.

Taken together, these findings support a consistent view of human hypothesis testing. Rather than arguing for biases in judgments or an insensitivity to disconfirming information, decisions in the present study suggest the operation of sensible reasoning processes. Students employ direct disconfirmation in a simple assessment task and counterfactual inference when they have to generate and assess hypotheses. In a sense, student reasoners operate both with Popper's (1972) requirements for a single test of a hypothesis and with Platt's (1964) heuristics for a programmatic approach to hypothesis testing.

We feel that the present analysis offers a description of the hypothesis testing strategies of successful and unsuccessful hypothesis testers, and provides a useful framework for creating and evaluating instructional programs for promoting scientific-like reasoning.

\section{REFERENCES}

Anderson, J. R. (1987). Methodology for studying human knowledge Behavioral \& Brain Sciences, 10, 467-505.

Baron, J. (1985). Rationality and intelligence. Cambridge: Cambridge University Press.

Bowers, K. S. (1977). Science and the limits of logic: A response to the Mahoney-DeMonbreun paper. Cognitive Therapy \& Research, 1, 239-246.

Bruner, J. S., Goodnow, J. J., \& Austin, G. A. (1956). A study of thinking. New York: Wiley.

CoHen, J. (1981). Can human irrationality be experimentally demonstrated? Behavioral \& Brain Sciences, 4, 317-370.

Ericsson, K. A., \&imon, H. A. (1980). Verbal reports as data. Psychological Review, 87, 215-251.

Evans, J. ST. B. (1982). The psychology of deductive reasoning. London: Routledge \& Kegan Paul.

Gorman, M. E. (1986). How the possibility of error affects falsification on a task that models scientific problem solving. British Journal of Psychology, 77, 85-96.

Gorman, M. E., \& Gorman, M. E. (1984). A comparison of disconfirmatory, confirmatory and control strategies on Wason's 2-4-6 task Quarterly Journal of Experimental Psychology, 36a, 629-648.

Gorman, M. E., Gorman, M. E., Latta, R. M., \& Cunningham, G. (1984). How disconfirmatory, confirmatory and combined strategies affect group problem solving. British Joumal of Psychology, 75 65-79.
Gorman, M. E., Stafford, A., \& Gorman, M. E. (1987). Disconfirmation and dual hypotheses on a more difficult version of Wason's 2-4-6 task. Quarterly Journal of Experimental Psychology, 39a, 1-28.

Hardin, C. L. (1980). Rationality and disconfirmation. Social Studies of Science, 10, 509-514.

HoRNBY, P. A. (1974). Surface structure and presupposition. Joumal of Verbal Learning \& Verbal Behavior, 13, 530-538

Klahr, D., Dunbar, K. (1988). Dual space search during scientific reasoning. Cognitive Science, $12,1-48$

Klayman, J., Ha, Y. (1987). Confirmation, disconfirmation and information in hypothesis testing. Psychological Review, 94, 211-228.

Mahoney, M. J. (1979). Psychology of the scientist: An evaluative review. Social Studies of Science, 9, 349-375.

Mahoney, M. J. (1980). Rationality and authority: On the confusion of justification and permission. Social Studies of Science, 10, 515-518.

Mahoney, M. J., \& DeMonbreun, B. G. (1977). Psychology of the scientist: An analysis of problem-solving bias. Cognitive Therapy \& Research, 1, 229-238.

MITROFF, I. (1974). The subjective side of science. New York: Elsevier Mynatt, C. R., Doherty, M. E., Tweney, R. D. (1977). Confirmation bias in a simulated research environment: An experimental study of scientific inference. Quarterly Journal of Experimental Psychology, 29, 85-95.

Mynatt, C. R., Doherty, M. E., Tweney, R. D. (1978). Consequences of confirmation and disconfirmation in a simulated research environment. Quarterly Journal of Experimental Psychology, 30, 395-406.

Platt, J. R. (1964). Strong inference. Science, 146, 347-353.

POPPER, K. R. (1972). Objective knowledge. Oxford: Oxford University Press.

RESCHER, N. (1964). Hypothetical reasoning. Amsterdam: North Holland.

Revlis, R., \& Hayes, J. R. (1972). The primacy of generalities in hypothetical reasoning. Cognitive Psychology, 3, 268-290.

TsCHIRGI, J. E. (1980). Sensible reasoning: A hypothesis about hypotheses. Child Development, 51, 1-10.

TUKEY, D. D. (1986). A philosophical and empirical analysis of subjects' modes of inquiry in Wason's 2-4-6 task. Quarterly Journal of Experimental Psychology, 38a, 5-33.

Tweney, R. D., Doherty, M. E., Worner, W. J., Puske, D. B., Mynatt, C. R., Gross, K. A., Arkkelin, D. L. (1980). Strategies of rule discovery in an inference task. Quarterly Journal of Experimental Psychology, 32, 109-123.

WASON, P. C. (1960). On the failure to eliminate hypotheses in a conceptual task. Quarterly Joumal of Experimental Psychology, 12, 129-140.

WASON, P. C. (1962). Reply to Wetherick. Quanerly Journal of Experimental Psychology, 14, 250.

WASON, P. C. (1968). On the failure to eliminate hypotheses: A second look. In P. C. Wason \& P. N. Johnson-Laird (Eds.), Thinking and reasoning. Middlesex: Penguin Books.

WEIMER, W. (1977). Scientific inquiry, assessment, and logic: Comments on Bowers and Mahoney-DeMonbreun. Cognitive Therapy \& Research, 1, 247-255.

WETHERICK, N. E. (1962). Eliminative and enumerative behavior in a conceptual task. Quarterly Journal of Experimental Psychology, 14, 246-249.

\section{APPENDIX A \\ Negative Outcome Story Problem}

Mark was going for a job interview, but he wanted to try a new approach. So:

- he gave concise answers to questions rather than long explanations

- he wore a suit rather than just a sportshirt and slacks

- he arrived for the interview fifteen minutes early rather than right on time

The interview went badly, it was brief and unproductive. 
Mark thought that the reason his interview went badly was the concise answers he gave. He thought that wearing a suit or arriving fifteen minutes early really didn't make any difference.

What should he do to prove this point?

a. On his next interview, he can give longer explanations, wear just a sportshirt and slacks, and arrive right on time. (CA)

b. On his next interview, he can still give concise answers, but wear just a sportshirt and slacks, and arrive right on time. (HOTAT)

c. On his next interview, he can give longer explanations rather than concise answers, and still wear a suit, and arrive fifteen minutes early. (VOTAT)

\section{APPENDIX B \\ Rule Discovery Instructions}

\section{Replication Groups:}

You will be given three numbers which conform to a simple rule that $I$ have in mind. This rule is concerned with a relation between any three numbers and not with their absolute magnitude, i.e., it is not a rule like all numbers above (or below) 50 , etc.

Your aim is to discover this rule by writing down sets of three numbers, together with reasons for your choice of them. After you have written down each set, I shall indicate whether your numbers conform to the rule or not by making note of this outcome on the record sheet provided. There is no time limit but you should try to discover this rule by citing the minimum sets of numbers.

Remember that your aim is not simply to find numbers which conform to the rule, but to discover the rule itself. When you feel highly confident that you have discovered it, AND NOT BEFORE, you are to write this nule down and circle it on your record sheet.

\section{Explicit Groups:}

In this task we are interested in how students think "scientifically" in a simulated experimental setting. You will be given an initial observation, three numbers which conform to a simple rule that I have in mind. Your task is to discover this rule by generating a possible rule (a hypothesis) and testing it with your own set of three numbers.

Write down your guess about the rule together with your test triple of numbers on the record sheet. The only information I will provide is an indication on your record sheet whether or not your test triple conforms or does not conform to my rule.

My rule is concerned with a RELATION between any three numbers and not with their absolute magnitude; so it is not a rule like "all numbers above (or below) 50 ", etc. Keep in mind that this is an arbitrary rule, one from an infinite set of rules for which the given observation: $\{2,4,6\}$ is a conforming instance.

You may continue as long as you wish to propose guesses and tests of them with number triples. Continue until you have proven to yourself that you know what the rule is. Only then, AND NOT BEFORE, are you to write down this rule and CIRCLE it on your record sheet.

Remember that the instruction "to prove" means to show that your rule is the only true one.

\section{APPENDIX C Full-Format Problem}

Mark was going for a job interview, but he wanted to try a new approach. So:

- he gave concise answers to questions rather than long explanations

- he wore a suit rather than just a sportshirt and slacks

- he arrived for the interview fifteen minutes early rather than right on time

The interview went badly, it was brief and unproductive. Mark thought that the reason his interview went badly was the concise answers he gave. He thought that wearing a suit or arriving fifteen minutes early really didn't make any difference.

What should he do to prove this point?

On Mark's next interview he should:

(Circle three letters - one for each element)

\begin{tabular}{|c|c|c|c|c|}
\hline \multicolumn{2}{|c|}{ Element } & \multicolumn{2}{|c|}{ KEEP THE SAME } & (or) CHANGE \\
\hline \multicolumn{2}{|c|}{ Answer Type } & \multicolumn{2}{|c|}{$\begin{array}{l}\text { a. give concise } \\
\text { answers }\end{array}$} & d. give long explanations \\
\hline \multirow{2}{*}{\multicolumn{2}{|c|}{$\begin{array}{l}\text { Clothing } \\
\text { Arrival Time }\end{array}$}} & \multirow{2}{*}{\multicolumn{2}{|c|}{$\begin{array}{l}\text { b. 'wear a suit } \\
\text { c. arrive fifteen } \\
\text { minutes early }\end{array}$}} & e. wear sportshirt \& slacks \\
\hline & & & & f. arrive on time \\
\hline Note: & $\begin{array}{l}\text { VOT } \\
\text { HOT } \\
\text { XVO? } \\
\text { XHO } \\
\text { HOLI } \\
\text { CHAI }\end{array}$ & $\begin{array}{l}\Gamma= \\
\Gamma= \\
\mathrm{AT}= \\
\mathrm{AT}= \\
\mathrm{ALL}= \\
\text { GE ALL }=\end{array}$ & $\begin{array}{l}\{\mathrm{b}, \mathrm{c}, \mathrm{d} \\
\{\mathrm{a}, \mathrm{e}, \mathrm{f} \\
\{\mathrm{a}, \mathrm{c}, \mathrm{e} \\
\text { b, d, f } \\
\{\mathrm{a}, \mathrm{b}, \mathrm{c} \\
\mathrm{d}, \mathrm{e}, \mathrm{f}\end{array}$ & $\left\{\begin{array}{l}\text { or }\{a, b, f\} \\
\text { or }\{c, d, e\}\end{array}\right.$ \\
\hline
\end{tabular}

\section{APPENDIX D Neutral-Negative Outcome Story}

Anne listened to her friend talk about his efforts to get to class on time. Her friend had changed his morning routine:
- he rode the bus instead of his bicycle
- he made tea instead of coffee to drink
- he set his alarm to "buzzer" instead of "music"

The new routine may not have helped, he wasn't on time for class. Her friend thought that the reason his new routine may not have helped was that he rode the bus. He thought that the type of drink (tea or coffee) or type of alarm setting really didn't make any difference.

Anne told her friend that if he wants to prove this point:

a. He should try his routine again but ride his bicycle instead of the bus, and still make tea and set his alarm to "buzzer." (VOTAT)

b. He should try his routine again still riding the bus, but make coffee and set his alarm to "music." (HOTAT)

c. He should try his routine again but ride his bicycle, make coffee and set his alarm to "music." (CA)

(Manuscript received September 25, 1987; revision accepted for publication June 25,1988 . ) 